\title{
ECONOMÍA DE LA MOVILIDAD GEOGRÁFICA DE LA MANO DE OBRA EN MÉXICO
}

\author{
Michael J. Greenwood \\ $\mathrm{Y}$ \\ JERRY R. LADMAN * \\ Arizona State University
}

\section{INTRODUCCIÓN}

EN GENERAL, MÉxico es considerado uno de los países del tercer mundo más exitosos en la búsqueda de su desarrollo económico después de la II guerra mundial. Durante tres decenios, después de 1940, el producto nacional bruto (PNB) de México en términos reales, creció a una tasa media anual superior al $6 \% .{ }^{1}$ Con esas tasas de crecimiento acelerado podría esperarse que la fuerza de trabajo del país se reubique tanto en términos ocupacionales como geográficos, en respuesta a los cambios en la estructura económica del país. En el decenio de 1960 casi el 10\% de la población del país cambió su lugar de residencia. ${ }^{2} \mathrm{El}$ propósito de este trabajo es examinar los flujos de la población entre entidades ęn México durante el decenio de los sesentas para estimar la magnitud de la influencia de diversos factores sobre la migración interregional.

Este estudio constituye un aporte a la creciente literatura sobre migración en los países de menor desarrollo relativo. Es similar en orientación a los principales estudios recientes sobre migración interregional en Ghana efectuados por Beals, Levy y Moses [1]; en la India y Egipto por Greenwood [2], [4] y [3] respectivamente; en Venezuela por Levy y Wadycki [5]) y [6]; en Brasil por Sahota [12]; y en Colombia por Shultz [13]. Unikel, Ruiz y Lazcano [15], emplearon un modelo de regresión múltiple en su estudio de migración rural-urbana en México para el decenio de

* Los autores son profesores asociados de economía de la Universidad del Estado de Arizona. Esta investigación se realizó gracias al apoyo del Population and Reproduction Grants Branch, Center for Population Research, National Institute of Child Health and Human Development, United States Department of Health, Education and Welfare. Los autores agradecen a Barbara Bindenagle, Barbara Orr y Barry Siegel por su ayuda en la tabulación de los datos y a Stuart Hazelkorn por su asistencia en la programación para el proceso electrónico de los mismos.

1 Véase Víctor L. Urquidi [16; 2].

2 Esta cifra no explica un cambio de residencia dentro de una entidad, ni las defunciones de migrantes en el decenio, y por lo tanto subestima la migración total en el país. 
1950, pero limitan su análisis a los factores que explican la expulsión de migración rural y no a la migración en general.

Los resultados del presente estudio son útiles no sólo para explicar la migración reciente en México, sino también para proveer pruebas adicionales de la teoría convencional de la migración, y hacer comparaciones con estudios similares para otros países de menor desarrollo relativo. Como se muestra más adelante, los resultados de este análisis tienden a corroborar muchos de estos estudios recientes, aunque existen diferencias apreciables en particular, con respecto al ingreso en la entidad de origen.

Una ventaja de la presente investigación es la calidad de los datos del censo mexicano, que permiten el uso de variables definidas al comienzo del decenio de 1960 para explicar los flujos migratorios sucedidos en un período de diez años. La importancia de los factores que afectaron la migración durante el decenio puede estimarse al utilizar los datos del principio del período, con lo que resulta una mejoría en la especificación relativa del modelo respecto de aquellos estudios que utilizan datos de finales del período como variables explicativas. ${ }^{3}$

La unidad espacial utilizada en el estudio es la entidad o territorio. En el momento del censo de 1970 había 29 estados, 2 territorios y un Distrito Federal. ${ }^{4}$ Un territorio, Baja California, se eliminó del estudio por su lejana localización geográfica al final de la península con lo que virtualmente quedaba aislada por carretera del resto del país. De esta manera, no existía una forma adecuada de comparar la distancia por carretera de este territorio con otras entidades del país. En consecuencia existen 31 entidades de origen y 30 de destino o sea 930 observaciones sobre flujos migratorios.

\section{EL MODELO}

En este estudio la tasa de migración $\left[\frac{M_{i j}}{P_{i}}\right]$-la migración de la entidad $i$ de origen en la entidad $j$ de destino durante el período 1960-1969, dividido entre la población total de la entidad de origen en 1960 - se utiliza como la variable dependiente. ${ }^{5}$ La relación básica estimada es:

3 El hecho de que los modelos en los que se utilizan variables con datos del fin del período para explicar la migración anterior tengan un sesgo inherente simultáneo causa desconfianza dado que la migración puede afectar estas variables.

4 En lo sucesivo, para simplificar, cuando se hable en general, todas las unidades serán referidas como "entidades".

5 Todos los datos utilizados en este estudio se tomaron del Censo General de Población de 1960 [8] y 1970 [9] de México; con las siguientes excepciones: la longitud en millas de la variable distancia se tomó del mapa oficial de carreteras publicado por el Departamento de Turismo en 1973; el dato de ingreso de 1960 se tomó de la Dirección General de Estadística [10]. 
$\left[\frac{M_{i j}}{P_{i}}\right]=\mathrm{f}\left(D_{i j}, Y_{i}, Y_{i}, U_{i}, U_{i}, P_{i}, P_{j}\right.$, errores aleatorios $)$

donde: $\quad D_{i j}=$ distancia por carretera, en kilómetros, entre las ciudades capitales de las entidades $i$ y $j$;

$Y_{i}, Y_{i}=$ la mediana de los salarios mensuales de los miembros de la fuerza de trabajo que declararon ingresos en el mes de mayo de 1960 en las entidades $i$ y $j$;

$U_{i}, U_{j}:=$ porciento de la fuerza de trabajo desempleada en las entidades $i$ y $j$ en 1960; y

$P_{i}, P_{j}=$ población total de las entidades $i$ y $j$ en 1960 .

La hipótesis subyacente en el modelo es que los individuos evalúan las oportunidades en diversas entidades y escogen su lugar de residencia sobre la base de "maximizar" su satisfacción. Así, una persona migraría de la entidad $i$ a la entidad $j$, si percibe que su satisfacción total será mayor en la entidad $j$ que en la entidad $i$ y que en todos los destinos alternativos, una vez descontados los riesgos y la incertidumbre. En esencia, el modelo establece que la tasa de migración depende de las oportunidades y características económicas en las entidades de origen y destino. En seguida se hace una descripción breve de las variables.

\section{Tasa de migración}

La variable dependiente, la tasa de migración $\left(M_{i j} / P_{i}\right)$, debe ser interpretada como la probabilidad de que una persona que residía en una entidad $i$ en 1960 emigrara a una entidad $j$ específica durante el período 1960-1969.

\section{Distancia}

La distancia de la movilización se ha cimentado de manera consistente como una variable muy importante en la explicación de la ubicación espacial de los migrantes en los países de menor desarrollo relativo. Nuestra variable distancia $\left(D_{i j}\right)$ se considera que expresa los costos monetarios y no monetarios de la movilización. ${ }^{6}$ Los primeros incluyen los desembolsos perdidos tanto como los costos de oportunidad asociados con la movilización. Los últimos son costos físicos asociados con la movilidad al desligarse de la familia, de amigos y familiares circunvecinos. Es probable quel entre mayor sea la distancia entre dos lugares menor sea el flujo de información entre ellos y en consecuencia será mayor el grado

6 Un buen comentario sobre los efectos de la distancia sobre la migración puede verse en Levy y Wadycki [7]. 
de incertidumbre asociado con una movilización entre ellos. Se supone entonces que la variable distancia tendrá un coeficiente negativo.

\section{Ingreso}

Se establece como hipótesis que los factores más importantes que afectan la migración son los niveles de ingreso en las entidades de origen y de destino. ${ }^{7}$ Se espera que los migrantes tenderán, ceteris paribus, a moverse de las entidades con ingresos relativamente bajos a entidades con ingresos relativamente elevados. En este estudio se utiliza la mediana de los salarios mensuales medios para las diversas entidades como representativo de la expectativa de ingresos de los migrantes potenciales. ${ }^{8}$

Se estableció como hipótesis que, ceteris paribus, entre más baja sea la mediana de ingresos mayor será el atractivo para migrar y entre mayor sea la mediana de ingreso menor será el atractivo para migrar.

\section{Desempleo}

Un migrante potencial puede considerar, ceteris paribus, que su posibilidad de obtener un trabajo es más baja entre más grande sea la tasa de desempleo prevaleciente en la localidad. Por lo tanto, se supone que entre mayor sea la tasa de desempleo en una entidad, mayor será la tendencia a emigrar y menor la tendencia a inmigrar a dicha entidad.

\section{Población}

La población de una entidad sive como indicador del tamaño del mercado de la fuerza de trabajo. ${ }^{9}$ Entre mayor sea la población, mayor será el número y tipo de oportunidades del trabajo disponibles. También se espera que entre mayor sea la población en las entidades de destino mayor sería la probabilidad de que las personas de una entidad de origen dado hayan migrado a esta entidad en el pasado. Los migrantes del pasado aumentan el flujo de información entre dos entidades y ello tiende a aumentar la migración entre entidades. ${ }^{10}$ Se supone que, ceteris paribus,

7 La mayoría de las teorías de migración consideran el valor actual de los beneficios esperados de la inversión en migración como la variable de ingreso adecuada (véase Sjaastad [14]. La teoría del valor actual es desarrollada en el contexto de la conducta individual. En este trabajo las limitaciones de información obligan a trabajar con la mediana del ingreso. Esto implica que todos los beneficios y los costos de la migración se acumulan en el período corriente y que la mediana de los ingresos es una buena apreciación de los futuros salarios esperados.

8 Esto implica que la nómina de salarios entre las diversas entidades es uniforme. La tendencia de la estructura de salarios es similar entre regiones como se muestra en Reynolds y Taft [11].

9 Véase Beals, Levy y Moses [1; 847].

10 Por ejemplo véase Greenwood [3; 287]. 
entre mayor sea la población en la entidad de destino mayor será la expectativa de migrar a estas entidades de cualquier entidad dada.

El resultado esperado en relación a la población de la entidad de origen es menos claro. Entre mayor sea la población de la entidad de origen es probable que el número de personas que han migrado de tal entidad a cualquier entidad de destino dada sea mayor. ${ }^{11}$ Sin embargo, esto no es muy claro dado que el número de migrantes aumentará en proporción a, en proporción mayor a, o en proporción menor a los aumentos de población en la entidad de origen.

\section{LOS RESUlTADOS}

Los coeficientes de la ecuación (1) se estimaron por mínimos cuadrados ordinarios y se ajustó una ecuación doble logarítmica para inter-

Cuadro 1

México: Migración entre entidades federativas. Coeficientes DE REGRESIÓN LOGARÍTMICA $(\beta)$ Y RAZONES $t$.

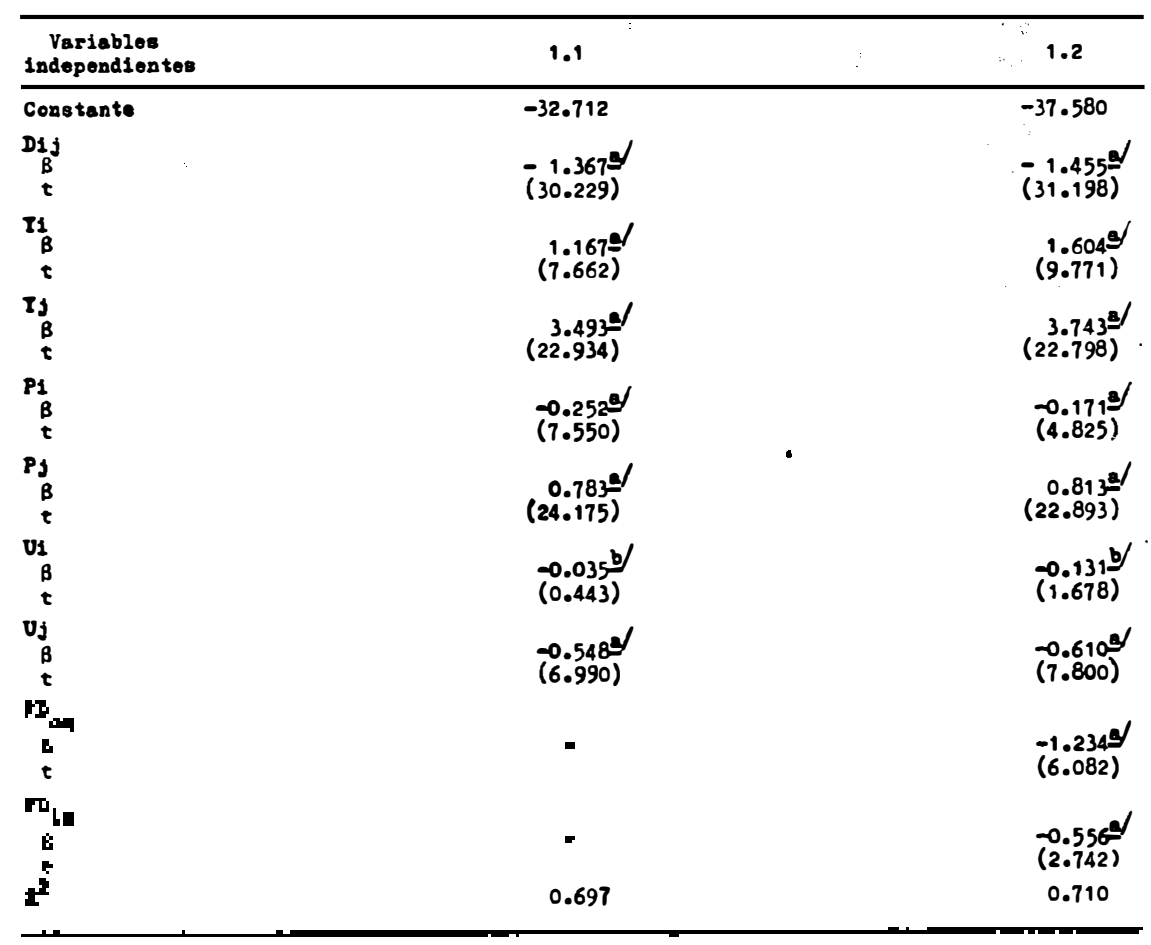

Significativos al nivel de 0.01 .

b Significativos al nivel de 0.05 .

11 Ibid., p. 287. 
pretar de manera directa tanto los coeficientes estimados como las elasticidades. Con el fin de tratar de determinar si otros efectos no considerados estaban asociados de manera específica con poblaciones muy numerosas de algunos países y la existencia de ciudades capitales importantes, se incorporaron variables dummy a un modelo alternativo para captar estos efectos en forma conjunta, en caso de que existiera.

Los resultados (1.1) de la estimación de la ecuación (1) y la alternativa (1.2) se presentan en el cuadro 1 . El cuadro 2 contiene los coeficien-

Cuadro 2

COEFICIENTES DE CORRELACIÓN SIMPLE

\begin{tabular}{|c|c|c|c|c|c|c|c|c|c|}
\hline & $\frac{n_{1,2}}{P 1}$ & Dif & I1 & $\mathbf{I j}$ & P1 & $\mathbf{P j}$ & U1 & נט & PD1. \\
\hline D1J & -0.493 & & & & & & & & \\
\hline $\mathbf{X}_{1}$ & 0.047 & 0.235 & & & & & & & \\
\hline $\mathbf{X}_{\mathbf{j}}$ & 0.314 & 0.235 & -0.033 & & & & & & \\
\hline Pi & -0.050 & -0.185 & -0.066 & 0.002 & & & & & \\
\hline$P j$ & 0.508 & -0.185 & 0.002 & -0.066 & -0.033 & & & & \\
\hline U1 & 0.110 & 0.018 & 0.708 & -0.024 & 0.042 & -0.001 & & & \\
\hline$U_{j}$ & 0.262 & 0.018 & -0.024 & 0.708 & -0.001 & 0.042 & -0.033 & & \\
\hline FD1 I & 0.000 & -0.127 & 0.331 & -0.011 & 0.356 & -0.012 & 0.187 & -0.006 & \\
\hline FDOM & 0.375 & -0.127 & -0.011 & 0.331 & -0.012 & 0.356 & -0.006 & 0.187 & -0.033 \\
\hline
\end{tabular}

tes de correlación simple asociados con todas las variables utilizadas en el estudio. El coeficiente de determinación múltiple $\left(R^{2}\right)$ es bastante elevado en ambas relaciones (0.697 y 0.710 , respectivamente). Todos los coeficientes estimados son significativos al nivel de $1 \%$ o aún menos, excepto el origen del desempleo. A continuación se comentan los resultados específicos para cada variable. Los comentarios se refieren a la regresión (1.1), a menos que se indique otra cosa.

\section{Distancia}

El coeficiente distancia se ha supuesto con signo negativo y es altamente significativo. Es claro que la distancia desalienta de manera drástica la migración en México. Estos resultados son muy parecidos a los de otros estudios de migración en países de menor desarrollo relativo. En estos estudios la variable distancia fue también negativa y de alta significación. La magnitud del coeficiente distancia para ecuaciones comparables tiende a ser ligeramente mayor en México que en Egipto (Greenwood [3:286] y Venezuela (Levy y Wadycki [5:79]), ligeramente menor que en Ghana (Beals, Levy y Moses [1:484]), y considerablemente menor que en la India (Greenwood [2:140]). 


\section{Ingreso}

El coeficiente del ingreso en la entidad de destino es positivo y significativo. Esto sugiere, como se suponía, que los migrantes tienden a movilizarse a entidades de ingresos elevados. Este resultado es semejante a los de otros estudios realizados en países de menor desarrollo.

El coeficiente del ingreso en las entidades de origen es también positivo y significativo, lo cual sugiere que mientras más grande sea el ingreso, más elevada será la posibilidad de que se efectúe la emigración en la entidad. De acuerdo con nuestra hipótesis el signo es irregular, y el resultado es diferente de manera sistemática con los hallazgos para otros países de menor desarrollo. ${ }^{12}$ Sin embargo, nuestros resultados coinciden con los hallazgos de Unikel, et. al. [15:47] para la migración rural-urba na en México durante el período 1950-1960. ${ }^{13}$

Estos autores encontraron que la emigración rural tiende a ser mayor en aquellas entidades con un elevado nivel de productividad agrícola y por lo tanto con un elevado nivel de ingresos. Una posible explicación de este fenómeno observado puede ser que las personas que viven en entidades de ingresos más elevados tienen una mayor disposición a la movilización debido al aumento en la información acerca de oportunidades de empleo alternativas que implican mayor remuneración y actividades económicas orientadas al mercado. Por ejemplo, los agricultores comerciales o trabajadores de granja estarían más en contacto con el "mundo exterior" que los tradicionales.

\section{Desempleo}

La tasa de desempleo en las entidades de destino influye sobre la migración. De acuerdo con la hipótesis establecida, entre mayor sea la tasa de desempleo en las entidades de destino, mayor será el desaliento para inmigrar a estas entidades. Este es otro indicador de que los migrantes se movilizan a lugares donde existen mejores perspectivas de empleo. Pocos de los otros estudios para países de escaso desarrollo incluyen esta variable, pero nuestros resultados son consistentes con éstos. ${ }^{14}$

El coeficiente para la tasa de desempleo en las entidades de origen no son significativamente diferentes de cero excepto cuando se emplean las variables dummy para el Distrito Federal. En la regresión que incluye las variables dummy, la elasticidad del desempleo en las entidades de ori-

12 Por ejemplo véase Beals, Levy y Moses [1], Greenwood [3; 286], [2; 144], Sahota $[12 ; 230]$ y Levy y Wadycki $[5 ; 79]$.

13 Greenwood $[4 ; 255]$ encontró un resultado similar para la migración ruralurbana en la India.

14 Vẻase Levy y Wadycki $[5 ; 79]$. 
gen, es no significativa hasta un nivel de más del $5 \%$ pero es de signo negativo, en contra de lo que se estableció como hipótesis. ${ }^{15}$ La explicación podría ser que el desempleo tiende a darse a edades muy jóvenes o en la vejez y entre grupos menos informados, los que tienden a tener una menor movilización.

\section{Población}

El coeficiente de la variable población en las entidades de destino es significativo y con signo positivo, como se esperaba. Sin embargo, el tamaño del coeficiente señala que el influjo a la entidad de destino es menor en proporción al crecimiento de la población de la entidad. ${ }^{18} \mathrm{El}$ coeficiente para la población de la entidad de origen es negativo y significativo. Esto sugiere que la emigración se incrementa también menos, en proporción que la población. ${ }^{17}$ Los resultados para las variables de población contrastan con los de otros estudios sobre migración para algunos

15 Levy y Wadycki $[5 ; 79]$ también encontraron este resultado para hombres jóvenes en Venezuela, pero no para hombres de mediana edad ni para ninguna clasificación de mujeres.

16 Otros estudios tienden a obtener resultados similares pero algunas veces $\epsilon 1$ coeficiente de elasticidad es considerablemente pequeño, véase por ejemplo Beals, Levy y Moses $[1 ; 484]$.

17 Dado que la relación estimada es de forma loglineal la cual emplea la tasa de migración como una variable dependiente, es necesario sumar 1.000 a la estimación del coeficiente de la variable población en la entidad de origen para espe. cificar la proporcionalidad y posibilitar la medición del coeficiente cuando se utiliza el flujo de migración. Considere la ecuación del flujo migratorio:

$$
M_{i j}=\alpha D_{i j}^{B 1} P_{i}^{B 3} \ldots \mu
$$

entonces:

$$
\ln M_{i j}=\ln \alpha+B_{1} \ln D_{i j}+B_{q} \ln P_{i}+B_{s} \ln P_{j}+\ldots+\ln \mu
$$

de manera alternativa, la ecuación de la tasa de migración es

$$
\begin{aligned}
& \frac{M_{i j}}{P_{i}}=\alpha D_{i j}^{B 1} P_{i}^{B 2} P_{i}^{B 3 \ldots \mu \mu} \\
& \ln M_{i j}-\ln P_{i}=\ln \alpha+B_{1} \ln D_{i j}+B_{2} \ln \dot{P_{i}}+B_{s} \ln P_{j}+\ldots+\ln \mu \\
& \text { agrupando términos } \\
& \ln M_{i j}=\ln \alpha+B_{1} \ln D_{i j}+\left(1+B_{g}\right) \ln P_{i}+B_{s} \ln P_{i}+\ldots+\ln \mu
\end{aligned}
$$

Así, dado que $B_{0}$ es el coeficiente estimado en el presente estudio, se debe agregar 1.000 a este coeficiente para obtener una medida de proporcionalidad. En el caso de la regresión $(1.1)$ el resultado es $(1-0.252)=0.748$ lo cual muestra que la migración es menor, en proporción, al nivel de la población de la entidad de origen. 
países de menor desarrollo, donde la variable población en las entidades de origen ha resultado significativa, pero de signo positivo. ${ }^{18}$

\section{Los efectos en el Distrito Federal}

En México, como es común en la mayoría los países de menor desarrollo relativo, el área de la capital —el Distrito Federal- sirve como centro de la actividad económica, gubernamental, política, social y cultural del país. ${ }^{19}$ En consecuencia, las variables dummy $-F D_{\text {im }}$ y $F D_{o m}$ - se utilizaron en la regresión 1.2 para aislar otros efectos no especificados que podrían concernir a la inmigración y a la emigración del Distrito Federal. Se ha establecido como hipótesis que, ceteris paribus, factores tales como esparcimiento cultural y social, mayores oportunidades educativas y la ubicación del asiento del gobierno federal, altamente centralizado, podrían ser algunos de los Iageares no especificados que ten-

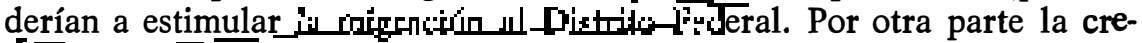
cient" contaminación, congestionamiento y el elevado costo de la vida pueden ser factores que alentarían la emigración.

En ambas ecuaciones estimadas, los coeficientes de las dos variables dummy fueron negativos y significativos. Si se considera al Distrito Federal como la entidad de origen, esto sugiere una fuerte renuencia a abandonar el Distrito, una vez radicado ahí. La "significancia" negativa puede reflejar la influencia de atracción de los factores culturales y sociales favorables asociados con la residencia en la ciudad de México, así como el hecho de que el comercio y la industria han tendido a concentrarse en el Distrito Federal debido a la presencia del gobierno y a las economías externas.

En contraste, el signo negativo de la variable dummy para la inmigración sugiere que en relación a otras entidades el Distrito Federal no fue particularmente atractivo para los migrantes. Quizá esto se debe al costo de la vida relativamente elevado en el Distrito, al congestionamiento y a la contaminación. Es posible que la explicación más razonable sea que el Distrito Federal resulta tan congestionado que los inmigrantes decidieron ubicarse en uno de los suburbios circunvecinos localizados en las entidades vecinas del Estado de México o Morelos, más que en el propio Distrito Federal y mantener los beneficios del medio ambiente cosmopolita y en proximidad a las actividades económicas gubernamentales.

18 Véase por ejemplo: Beals, Levy y Moses [1]; Greenwood [2], [3]; y Wadycki [5].

19 En 1970, el Distrito Federal registró más del 12\% de la población del país. En el Censo General de Población de 1970 [9] se registraron 781987 personas que emigraron desde el Distrito Federal y 2385304 que inmigraron al Distrito Federal desde 1960. Así, el Distrito Federal registró casi el 30\% de los flujos de migración total en el país para este período. 


\section{RESUMEN Y CONCLUSIONES}

En este trabajo se diseñó un modelo de migración entre entidades en México y se estimó con información para el período 1960-1969. Las variables dependientes seleccionadas "explican" una proporción razonablemente elevada de las variaciones en la migración entre entidades. Todas las variables, excepto el desempleo en las entidades de origen son significativas al nivel del $1 \%$ o menos aún.

De acuerdo con la hipótesis establecida, los migrantes son en gran medida desalentados por la distancia. Todas las variables para las entidades de destino registraron coeficientes significantes y del signo supuesto. Se observó que las personas tienden a migrar a entidades más pobladas, a las de mayores ingresos y a las entidades con tasas de desempleo más reducidas. Estos resultados son consistentes de manera uniforme con los de estudios de otros países de menor desarrollo.

En contraste, la estimación del coeficiente de la variable ingreso en la entidad de origen registra un signo diferente al establecido como hipótesis, lo cual significa que entre mayor es el ingreso en la entidad de origen mayor es la emigración. Este resultado contrasta con la mayoría de los obtenidos para países de menor desarrollo. El parámetro para la población de las entidades de origen es también altamente significativo y negativo. La variable desempleo en las entidades de origen es no significativa en la ecuación que no incluye variables dummy.

Se estimaron ecuaciones con variables dummy para la emigración e inmigración del Distrito Federal para determinar si algunos factores no especificados influían sobre la migración hacia o desde este centro de actividad económica, política, industrial y social. Los coeficientes de ambas variables fueron significativos y negativos. Esto sugiere para la emigración una fuerte renuencia a abandonar el Distrito, lo cual es sostenible dado el elevado estandard de vida y los esparcimientos que ofrece la ciudad y al hecho de que constituye el centro de la actividad comercial, económica y gubernamental y de toma de decisiones. El coeficiente negativo para la variable inmigración es más difícil de explicar. Es posible que la explicación más realista es que el Distrito empieza a alcanzar su capacidad para absorber población dada su elevada densidad y, por lo tanto, los inmigrantes recientes al área han elegido en su lugar vivir en ambientes menos congestionados pero contiguos a la ciudad capital localizados en entidades vecinas.

La presión de la población sobre la disponibilidad de recursos es un factor importante que origina muchos de los problemas sociales, económicos y políticos en la mayoría de los países de menor desarrollo como México. En parte, estos problemas son originados o por lo menos intensificados por la migración. 
Así, los encargados de diseñar las políticas deben estar interesados en particular en los determinantes de la migración. Aun cuando este estudio es limitado en alcance debido a la falta de datos con la suficiente desagregación sobre las características de los migrantes, representa un intento de explicar estos determinantes para México. En la medida en que se disponga información más desagregada, modelos similares a los utilizados en este estudio serán de mayor utilidad para los diseñadores de políticas interesados en influir sobre la migración hacia (o desde) áreas particulares.

\section{Bibliografía}

1. Ralph E. Beals, Mildred B. Levy y Leon N. Moses, "Rationality and Migration in Ghana", The Review of Economics and Statistics, 49, noviembre de 1967, pp. 480-486.

2. Michael J. Greenwood, "An Analysis of the Determinants of Internal Labor Mobility in India", The Annals of Regional Science, V, junio de 1971, pp. 137-151.

3. Michael J. Greenwood, "The Determinants of Labor Migration in Egypt", Journal of Regional Science, 9, Núm. 2, 1969, pp. 283-290.

4. Michael J. Greenwood, "A Regression Analysis of Migration to Urban Areas of a Less- Developed Country: The Case of India", The Journal of Regional Science, II, Núm. 2, 1971, pp. 253-262.

5. Mildred B. Levy y Walter J. Wadycki. "A Comparison of Young and Middle-Aged Migration in Venezuela", The Annals of Regional Science, VI, diciembre de 1972, pp. 73-85.

6. Mildred B. Levy y Walter J. Wadycki, "Lifetime Versus one-Year Migration in Venezuela", Journal of Regional Science, 12, Núm. 3, 1972, pp. 407-415.

7. Mildred B. Levy y Walter J. Wadycki, "What is the Opportunity Cost of Moving? Reconsideration of the Effects of Distance on Migration", Economic Development and Cultural Change, 22, enero de 1974, pp. 198-214.

8. México, Secretaría de Industria y Comercio, Direción General de Estadística. VIII Censo general de población, 1960. Resumen general, México, 1962.

9. México, Secretaría de Industria y Comercio, Dirección General de Estadística, IX Censo general de población, 1970. Resumen general, México, 1972.

10. México, Secretaría de Industria y Comercio. Dirección General de Estadística, "Ingresos por trabajo de la población económicamente activa y jefes de familia", VIII Censo de población, 1960, México, 1964.

11. L. G. Reynolds y C. H. Taft, The Evolution of the Wage Structure, New Haven, Yale University Press, 1956.

12. Gian S. Sahota, "An Economic Analysis of Internal Migration in Brazil", Journal of Political Economy, 76, marzo-abril de 1968, pp. 218-245. 
13. T. Paul Schultz, "Rural-Urban Migration in Columbia", The Review of Economics and Statistics, LIII, mayo de 1971, pp. 164-168.

14. Larry A. Sjaastad, "The Costs and Returns of Human Migration", Journal of Political Economy, 70, octubre de 1962, suplemento, pp. 80-93.

15. Luis Unikel, Crescencio Ruiz Ch. y Omar Lazcano, "Factores de rechazo en la migración rural en México 1950-1960", DEMOGRAFía X ECONOMíA, VII, 1973, pp. 24-57.

16. Víctor L. Urquidi, "An Overview of Mexican Economic Development", Weltwirtschaftliches Archiv, 101, 1968, pp. 2-20. 